\title{
Computing the Future: Digital encounters in art and science when da Vinci meets Turing
}

\author{
Tula Giannini \\ Professor of Information \\ School of Information \\ Pratt Institute \\ New York, USA \\ https://sites.google.com/view/tgiannini/ \\ giannini@pratt.edu
}

\author{
Jonathan P. Bowen \\ Emeritus Professor of Computing \\ School of Engineering \\ London South Bank University \\ London, UK \\ http://www.jpbowen.com \\ jonathan.bowen@lsbu.ac.uk
}

\begin{abstract}
Computing the future, as life and research moves to the Internet, we are engaged increasingly in digital encounters from present to past and into the future with real people, events and documents. This paper focuses on the newly born-digital relationship between Alan Turing, father of computer science, and Leonardo da Vinci, master of Renaissance art and science - both revered as visionary geniuses, prophets of the future. Given the continued growth of digitised materials that are daily entering global consciousness, it is only relatively recently that both da Vinci's notebooks and paintings, and Turing's archive, are online and searchable. Thus we are able for the first time to relatively easily juxtapose and compare their work, and see that they have much in common in terms of what it means to human in science, art and the natural world, from da Vinci's in-depth studies of the mechanisms of the human body, mind, and soul, foundational to his art, and to Turing's discoveries in Artificial Intelligence (Al), machine learning, and morphogenesis. Considering their points of concurrence in the digital world brings into focus our global network of digital places and spaces, where science, art, and nature, including real and artificial life, become unbounded.
\end{abstract}

Digital art. Digital culture. Digital heritage. Digital media. Postdigital world. Alan Turing. Leonardo da Vinci.

\section{When Digital da Vinci meets Turing}

(by T. Giannini)

\author{
Touched by nature \\ new nomenclature \\ unknown destination \\ Seeking integration \\ of science and art \\ Da Vinci and Turing \\ writing in code \\ revealing secrets of art \\ and life à la mode \\ From inside out \\ What's life about \\ The process of creation \\ human instigation \\ Mirrors of the mind \\ identity undefined \\ real and artificial \\ feeling physical \\ It's still about \\ Love $\bigcirc$
} algorithms and computation traveling in a sea of zeros and ones

\section{INTRODUCTION}

Both Leonardo da Vinci (1452-1519) and Alan Turing (1912-1954) defined scientific and cultural revolutions that forever changed our ways of thinking and seeing the world, from the humanism of Renaissance life to $21^{\text {st }}$-century computational culture, to how we think about ourselves and what is means to be human having an individual voice and the freedom of self-expression (Zöllner 2003; Copeland et al. 2017). During the final years of his life, Turing went back to nature to study its processes (see Figures 1 and 2), focusing on morphogenesis, embryology, phyllotaxis (Scienceblocks 2018), neural networks, the human brain and how it thinks. Resetting the locus of his work to the botanical world. Along these lines da Vinci writes (MacCurdy 1955, Botany 307):

"All seeds have the umbilical cord which breaks when the seed is ripe. And in like manner they have matrix and secundina, as is seen in herbs and all the seeds which grow in pods. But those which grow in shells, such as hazel-nuts, 
pistachio-nuts and the like have the umbilical cord long, and this shows itself in their infancy."

Leonardo da Vinci's words allude to human gestation and to his lost painting of Leda and the Swan, showing Leda's children hatching from eggs (see Figures 3 and 4).

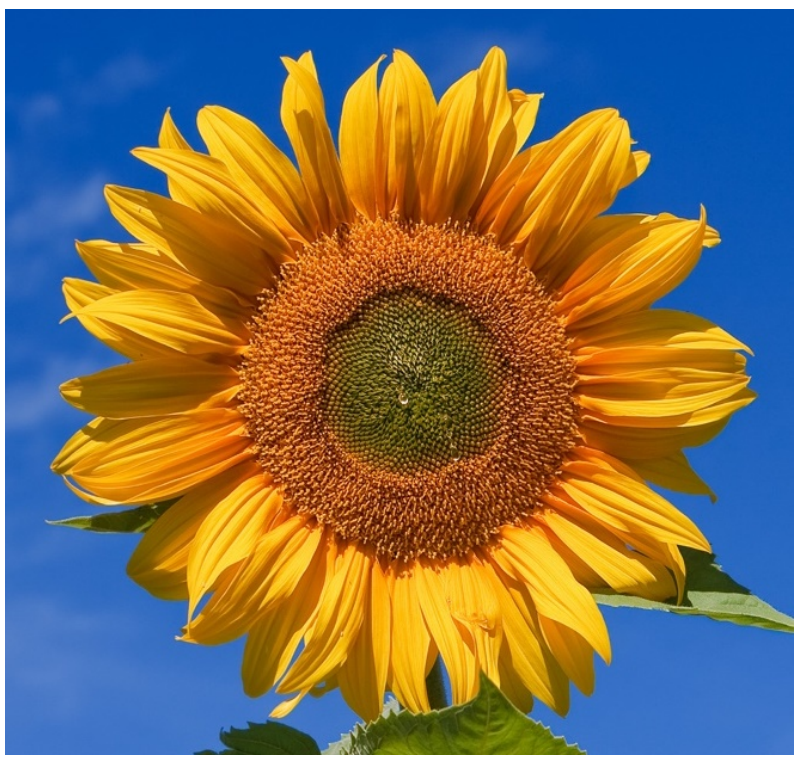

Figure 1: Sunflower in natural landscape. Wikimedia. https://commons.wikimedia.org/wiki/ File:Sunflower sky backdrop.jpg

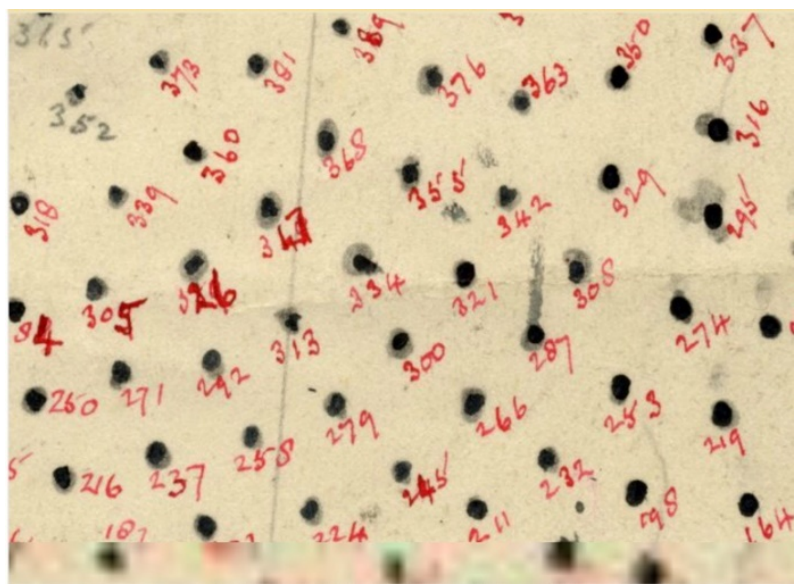

Figure 2: Detail from Phyllotaxis drawing by Alan Turing based on studies of the spiral patterns of sunflowers, 1953. The Turing Digital Archive, AMT/C/25. http://www. turingarchive.org/viewer/?id=142\&title=95

In the current supercharged digital environment, digital time accelerates the advance of digital culture, while Al embeds itself into every aspect of contemporary life from the boardroom to the bedroom. Our intense efforts to compute the future of the postdigital world, reveals the integration of the universal processes of the natural and computational worlds with the $0 \mathrm{~s}$ and $1 \mathrm{~s}$ of the global digital ecosystem - in this paper, we picture da Vinci and Turing sharing their vision.

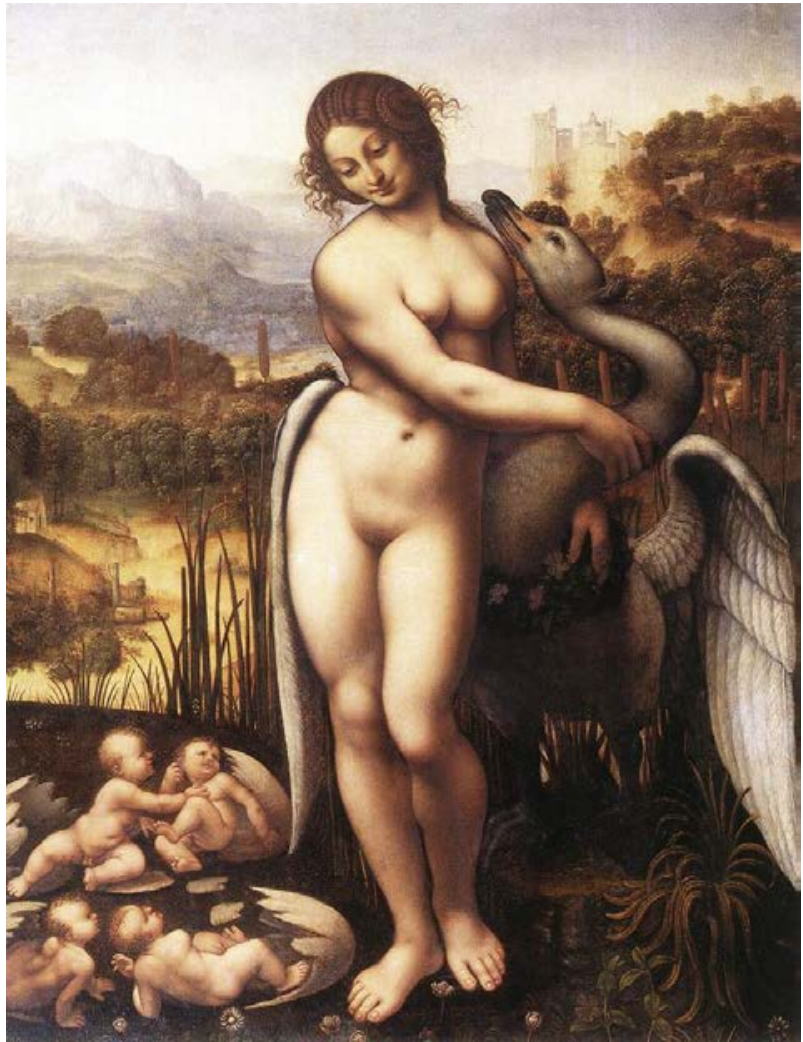

Figure 3: Leda and the Swan copy by Cesare da Sesto after a lost original by da Vinci, 1515-1520, Oil on canvas, Winton House, Salisbury, UK. Wikimedia. https://commons. wikimedia.org/wiki/ File:Leda and the Swan 1505-1510.jpg

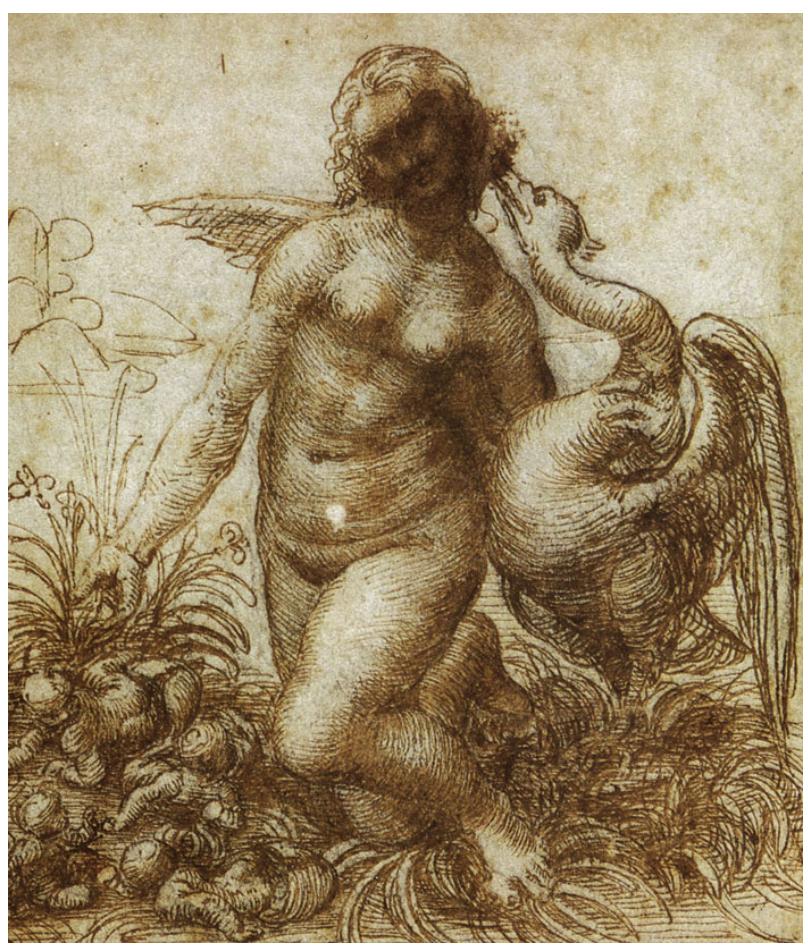

Figure 4: Leda and the Swan, drawing by Leonardo da Vinci, c.1507. Chatsworth House, UK. Wikimedia. https://commons. wikimedia.org/wiki/ File:Study for the Kneeling Leda.jpg 
Da Vinci's interest in the subject of Leda and the swan drawn from Greek Mythology, tests our understanding of human life and religion, and reveals the complexity of his perspectives and ability to interweave nature with real and artificial life. Da Vinci's original painting believed to be lost or perhaps destroyed, was a spectacular work inspiring copies by contemporary artists, sheds light on his views on sexuality, human consciousness and being. Today, the idea of human babies being born (or hatched) from eggshells might not seem farfetched, seeing that new technology has brought claims that an artificial womb for humans is already in development in the Netherlands (Devlin 2019).

Turing's digital computer work introduced thinking machines and artificial intelligence, and what is known as the Turing Test to compare machine and human intelligence (Turing 1950). Fast forward to 16 February 2011, when IBM's Watson computer won the Jeopardy TV gameshow beating the show's top two players, viewers were stunned Turing might have said, to be expected, and much more to come (Markoff 2011).

We see that da Vinci and Turing could mix the rigour of science and mathematics, with imagination and playfulness, bringing together real and artificial life, to make discoveries that continue to inspire and challenge our sense of being human, while being less sure about reality.

Discussing painting, Leonardo da Vinci writes (MacCurdy 1955, Comparison of the Arts 854):

"And this [painting] truly is a science and the true-born daughter of nature, since painting is the offspring of nature. But in order to speak more correctly we may call it the grandchild of nature; for all visible things derive their existence from nature, and from these same things is born painting. So therefore we may justly speak of it as the grandchild of nature and as related to God himself."

If as Leonardo da Vinci writes, that painting is "the grandchild of nature" and "related to God himself", then the painter would appear to be doing God's work.

\section{DA VINCI AND TURING COMPARED}

\subsection{Seeing Anatomy in Painting}

The drawings of Leonardo da Vinci expose his views and visualisations of the human body, and the complex internal anatomical systems supporting it, as is vividly revealed in this painting of St Jerome (see Figure 5). This work stands out for its anatomical nature which we see depicted in da Vinci's drawings of the shoulder (see Figure 6).

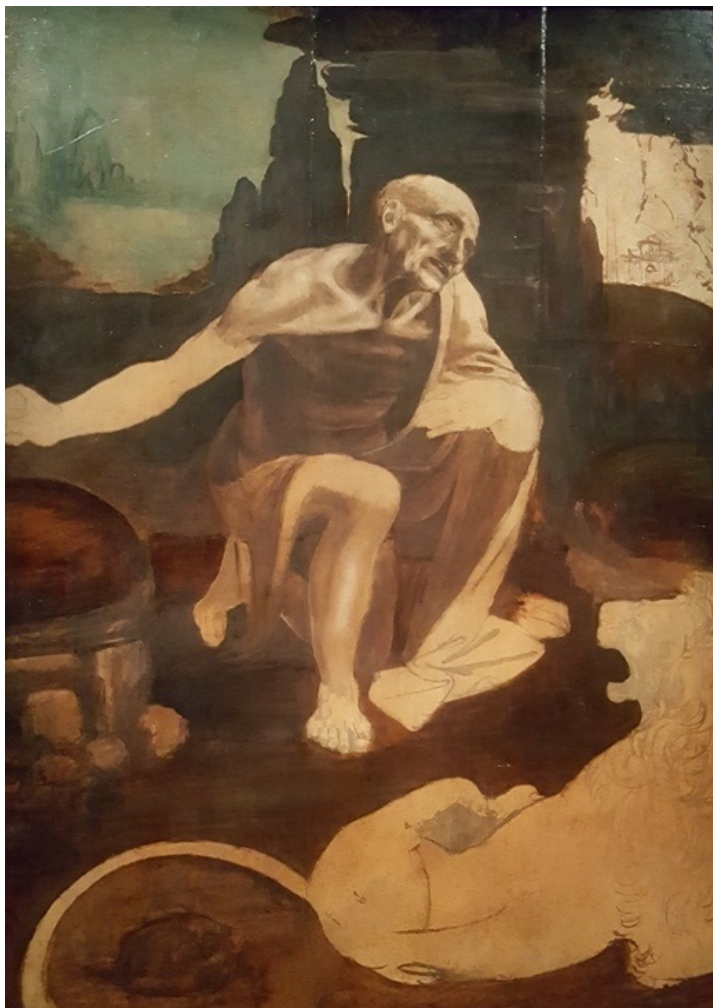

Figure 5: St Jerome Praying in the Wilderness by Leonardo da Vinci. Featured painting for the da Vinci exhibition at the Metropolitan Museum of Art, New York, October 2019. The painting was on loan from the Vatican to honour the 500th anniversary since da Vinci's death. Photograph by T. Giannini.

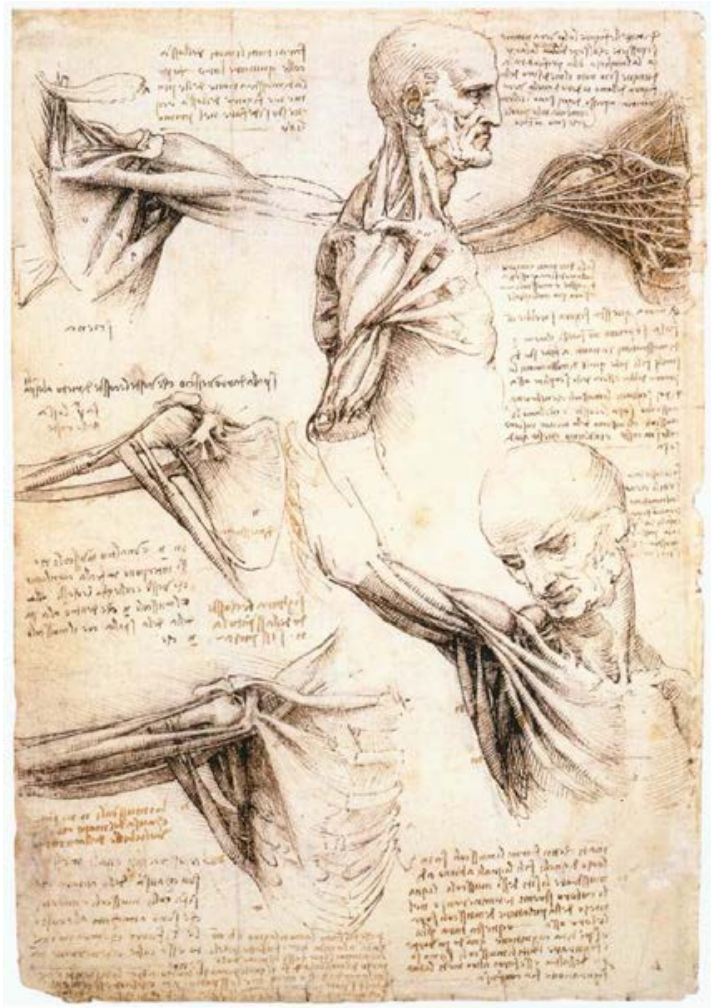

Figure 6: Anatomical studies of the shoulder, by Leonardo da Vinci. LeoonardoDaVinci.net. 
https://www.leonardodavinci.net/anatomical-studies-ofthe-shoulder.jsp

Another area of intense investigation for da Vinci is seen in his drawings of mechanical processes. His notebooks contain extensive material on flight inspired by the flight of birds (see Figure 7).

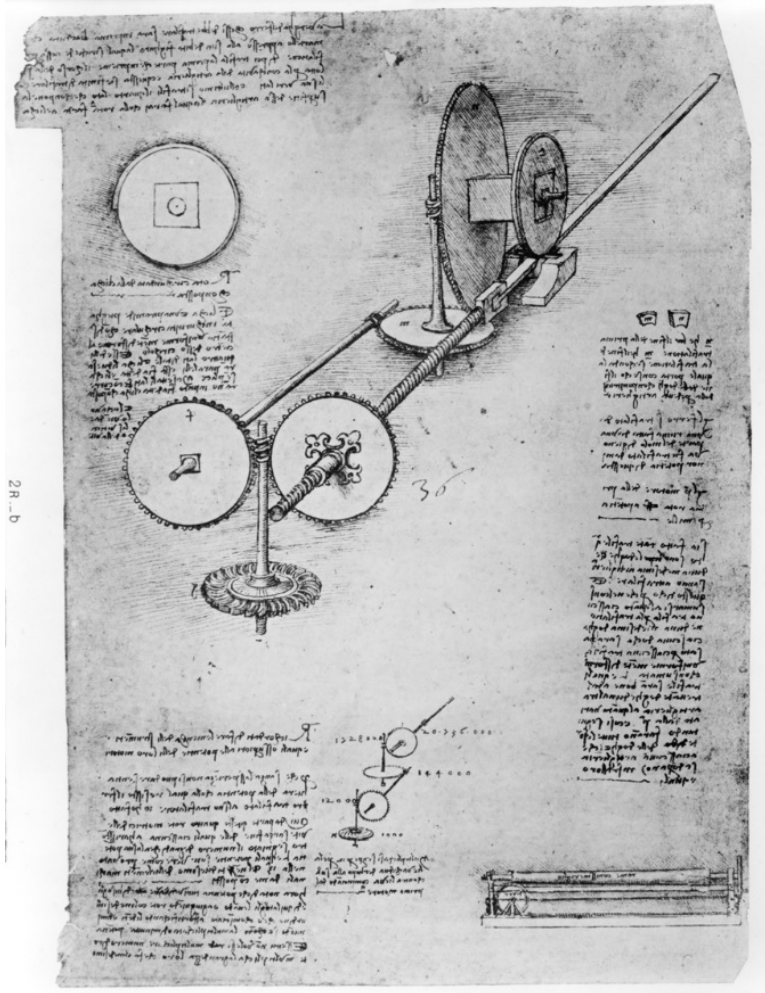

Figure 7: Mechanical wing. Library of Congress, USA. Wikimedia. https://commons. wikimedia.org/wiki/ File:Reproduction of page from notebook of Leonardo da Vinci showing the operation of a mechanical wing LCCN2006681096.jpg

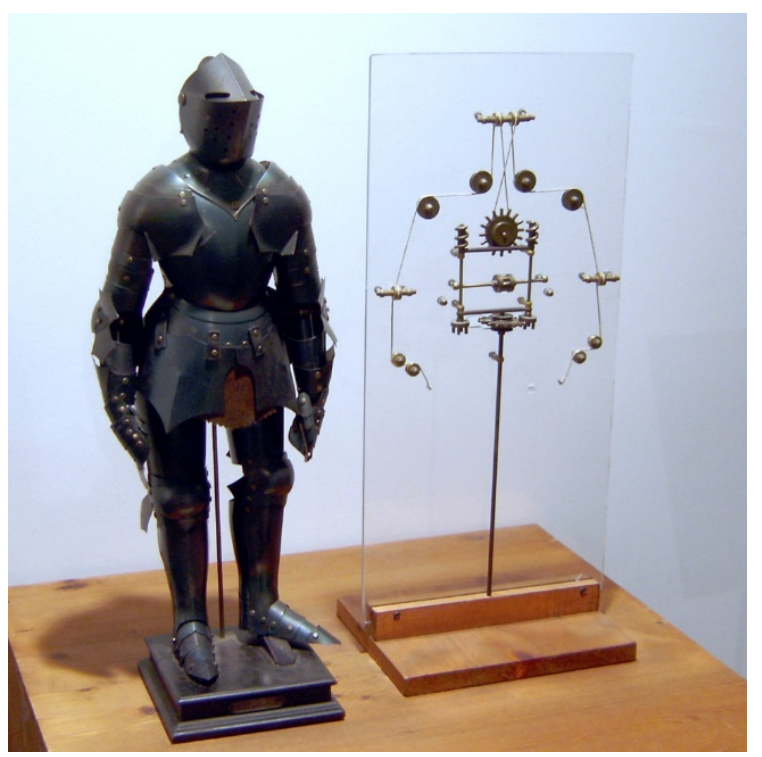

Figure 8: Model of a robot based on drawings by Leonardo da Vinci. Photograph by Erik Möller. "Leonardo da Vinci. Mensch - Erfinder - Genie" exhibit, Berlin, Germany, 2005. Wikimedia.
https://commons.wikimedia.org/wiki/File:LeonardoRobot3.jpg

The ACM A.M. Turing Award leads back to Turing's pioneering ideas in machine learning (Turing 1950; ACM 2018). In addition, recent research makes Al even more human when computers now can simulate the human senses - speak, see, read, hear, feel, etc. (Rharbaoui 2016). Turing meets da Vinci on the Internet - in that Turing's Al and machine learning makes computing more human and closer to natural interaction. Robots often simulate and imitate human capabilities and even physical attributes (see Figures 8 and 9).

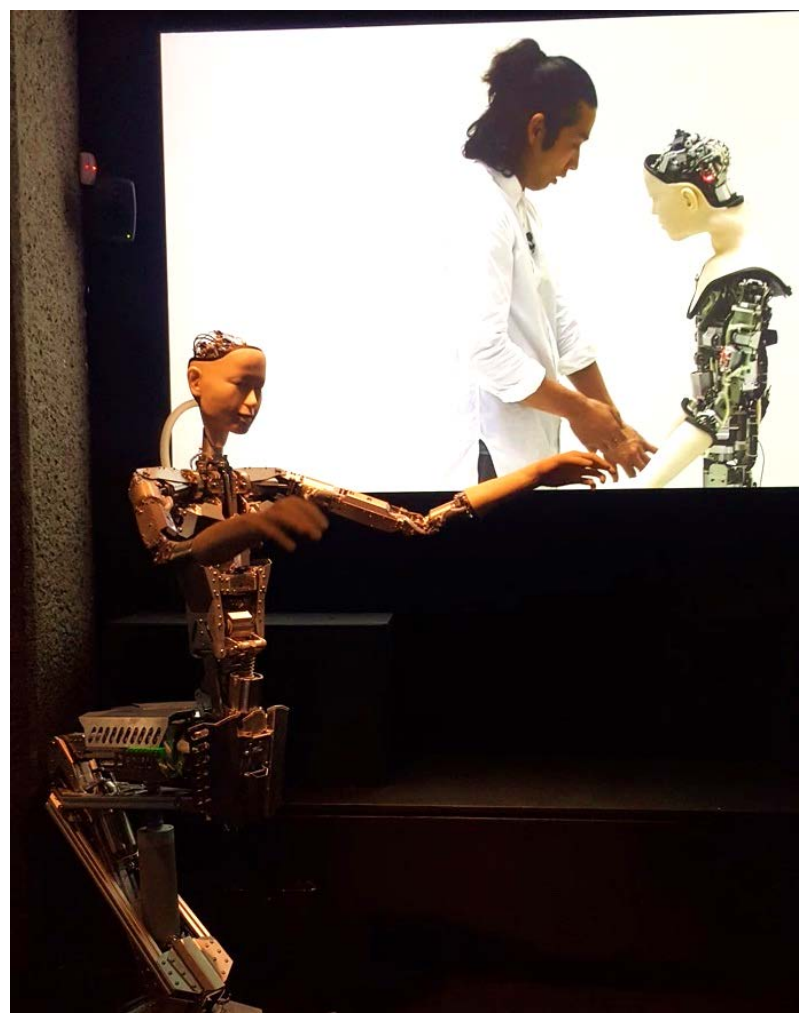

Figure 9: Artificial Life. The broad research field of artificial life studies and simulates processes in nature. Often a speculative field, it explores existing and future forms of life. A-life spans areas such as neural networks, themselves drawn natural processes, but also biomimicry, genomic and synthetic biology. "Al: More than Human" exhibition, Barbican, London, UK, 2019. Photograph by T. Giannini.

The ACM (2018) notes:

"In recent years, deep learning methods have been responsible for astonishing breakthroughs in computer vision, speech recognition, natural language processing, and robotics-among other applications. ... Deep neural networks are responsible for some of the greatest advances in modern computer science, helping make substantial progress on long-standing problems in computer vision, speech recognition, and natural language understanding ... By dramatically improving the ability of computers to make sense of the world, deep neural networks 
are changing not just the field of computing, but nearly every field of science and human endeavor."

\subsection{Beyond Nature in Painting and Machines that can Think}

Both da Vinci and Turing worked on machines with gears, producing remarkably similar diagrams (see Figures 10 and 11).

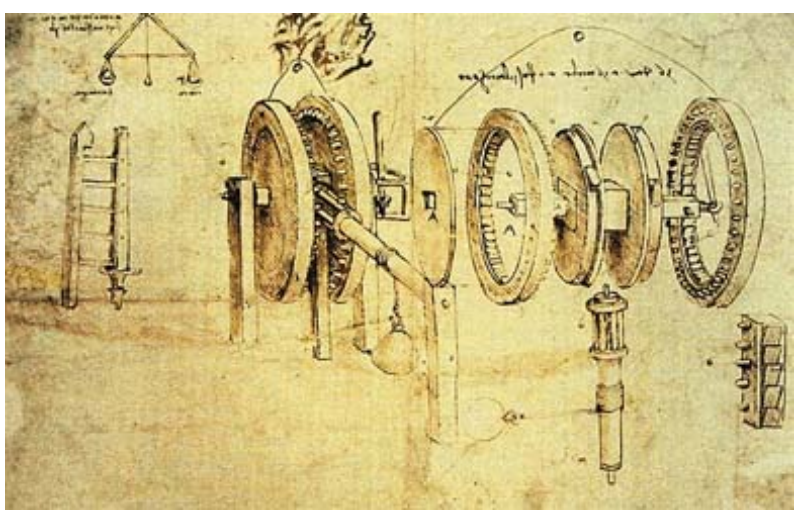

Figure 10: Drawing by Leonardo da Vinci. A geared device assembled and disassembled. Wikimedia. https://commons.wikimedia.org/wiki/File:Tрансорормаци я переменного движения в непрерывное.jpg

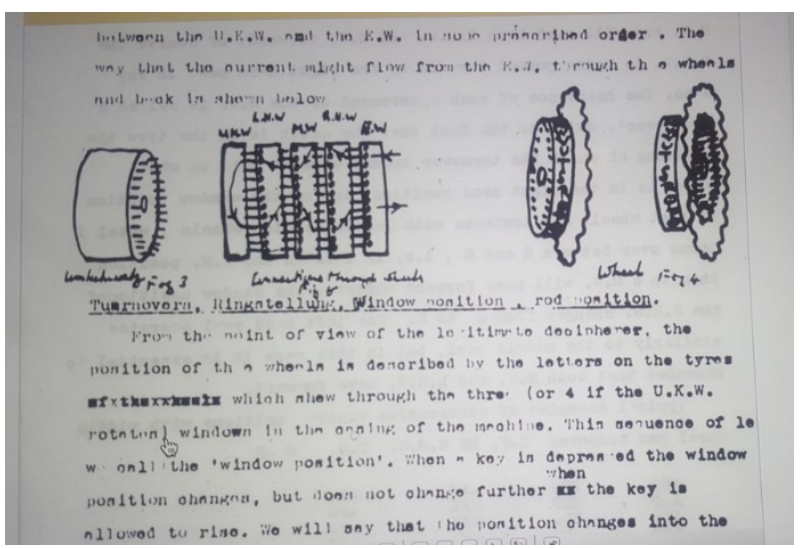

Figure 11: Detail from Turing's drawing of wheels and rings of the Enigma machine from his Treatise on the Enigma. c.1939-42, page 3 http://www.turingarchive.org/viewer/?id=147\&title $=03$ (Gladwin 1997).

On 15 May 1951, the BBC broadcasted a short lecture on the radio by Alan Turing. The talk included mention of germination, programming a machine to think, and many illusions to flowers and nature (Turing 1951).

From the Turing Digital Archive online, we see that Turing defines one of the key problems that we continue to address - "how to programme a machine to imitate the human brain, or as we might say more briefly, if less accurately, to think" (Turing Digital Archive n.d., AMT/B/5; Turing 1951). Leonardo da Vinci's version of this question was how to paint the human form that imitates nature while showing the life force of the body's mechanisms. Turing invokes Lady Lovelace's dictum: "the machine can do whatever we know we can order it to perform." To point to another direction, Turing (1951) asserts:

\begin{abstract}
"One does not need to be able to understand how these orders lead to the machine's subsequent behaviour, any more than one needs to understand the mechanism of germination when one puts a seed in the ground. The plant comes up whether one understands or not. If we give the machine a programme which results in its doing something interesting which we had not anticipated I should be inclined to say that the machine had originated something, rather than to claim that its behaviour was implicit in the programme."
\end{abstract}

He goes on to say that most people do not like the idea that machines can think because, "if a machine can think, it might think more intelligently than we do, and then where should we be?" Turing ends the BBC broadcast noting that, "the whole thinking process is still mysterious to us, but I believe that the attempt to make a thinking machine will help us greatly to find out how we think ourselves" (Turing Digital Archive n.d., AMT/B/5).

Turing's manner of interrogating his subject connects to da Vinci's process of thinking relying heavily on observation and experience learning from real life - seeing the human body as a machine controlled by the processes of nature of which we are not in control. While Turing sought to create a machine that imitated human thinking, da Vinci played the "Imitation Game" through his drawings and paintings penetrating the human mind in ways that go beyond the natural world to a heightened sense of feeling close connections to the essence of life. As a mirror reflecting life and the human mind, through the lens of these visionary geniuses, we can see into both the natural and artificial worlds we now inhabit.

\subsection{The Mind as a Mirror of Nature and Al and Machine Learning Mirroring the Human Brain}

Leonardo da Vinci recorded his thoughts on the "mind of the painter" as a mirror of nature to reflect and curate images (MacCurdy 1955, Painting 857):

"The mind of the painter should be like a mirror which always takes the colour of the thing that it reflects, and which is filled by as many images as there are things placed before it. Knowing therefore that you cannot be a good master unless you have a universal power of representing by your art all the varieties of the forms which nature produces, - which indeed you will not know how to do unless you see them and retain them in your mind, - look to it, O Painter, that when you go into the fields you give your attention to the various objects, and 
look carefully in turn first at one thing and then at another, making a bundle of different things selected and chosen from among those of less value."

Da Vinci goes on to explain (MacCurdy, 1955 Precepts 879):

"How the mirror is the master of painters - When you wish to see whether the general effect of your picture corresponds with that of the object represented after nature, take a mirror and set it so that it reflects the actual thing, and then compare the reflection with your picture, and consider carefully whether the subject of the two images is in conformity with both, studying especially the mirror."

Mirrors often appear in painting and for portrait paintings mirrors are used by artist to paint a reflection of self (see Figure 12, where the mirror in the hand looks remarkably like a smartphone). Contemporary artists use the smartphone camera to create a myriad of selfie images from which they curate identity and convey states of being (Manevskava 2017).

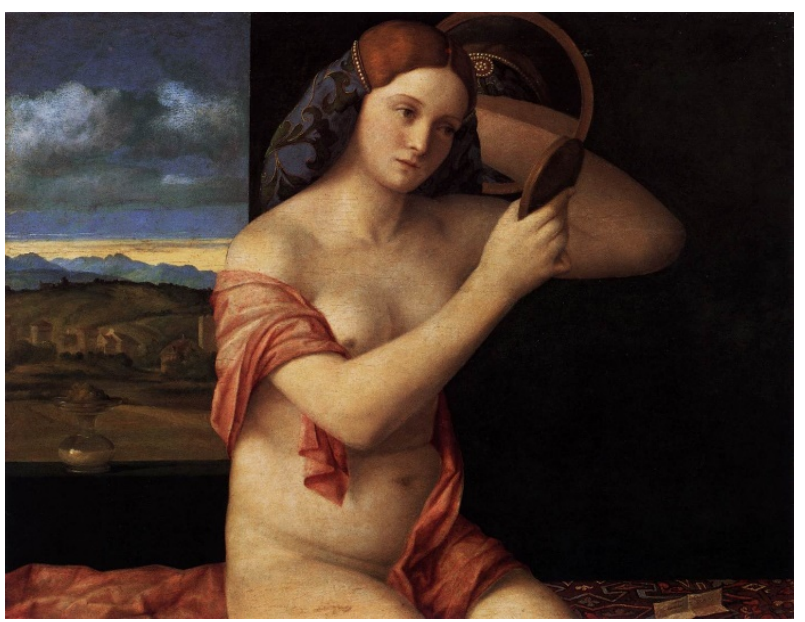

Figure 12: Nude young woman looking at herself in the mirror, by Giovanni Bellini (1430-1516), painted 1515. A Venetian artist, he was a contemporary of da Vinci. Wikimedia. https://commons. wikimedia.org/wiki/ File:Giovanni_Bellini - Naked Young Woman in Front of the Mirror - WGA1785.jpg

It is likely that Turing saw the digital computer representing a natural state of being in the universe derived from the natural phenomenon of ones and zeros and mathematical logic. Further consider that both da Vinci and Turing were mathematicians seeing mathematics as part of universal logic. In this context, the line between natural and artificial becomes blurred, as does synthetic and real.

As robots increasingly intrude into everyday life, one can detect contradictory states of human elation and fear as Turing notes, while images of robot invaders and artificial life seem to predominate in Hollywood productions. Leonardo da Vinci's studies of human anatomy are both admired yet many people cringe at the thought of dissection and seeing the internal structures and organs.

\subsection{Love and the Lover Imagined by da Vinci and Sung by Taylor Swift}

The topic of the lover is taken up by Leonardo da Vinci under the subject of philosophy (MacCurdy 1955, Philosophy 67):

\begin{abstract}
"The lover is drawn by the thing loved, as the sense is by that which it perceives, and it unites with it, and they become one and the same thing. The work is the first thing born of the union; if the thing that is loved be base, the lover becomes base. When the thing taken into union is in harmony with that which receives it, there follow rejoicing When the lover is united to that which is loved it finds rest there; when the burden is laid down there it finds rest."
\end{abstract}

Both da Vinci and Turing had male lovers, a crime for which they faced criminal prosecution. However, these consequences do not mitigate the role their lovers played in their ways of being human (Dowd 2014).

Fast forward to present, we see that the popular American songwriter, Taylor Swift (born 1989), takes up this subject in her 2019 album song entitled "Lover." Her lyrics capture the essence of the lover's desires (Swift 2019):

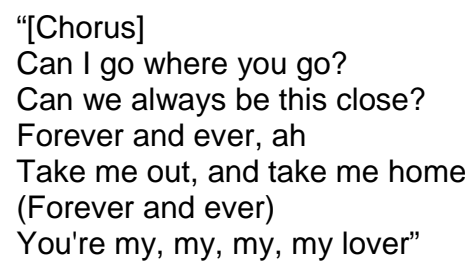

Digital encounters between Turing and da Vinci open up new connections between them, and new ways of understanding their work in which we see commonalities around science, art and technology. Importantly, their humanistic approach to life that pays tribute to Greek and Roman science and art. Both were pioneers in their field. While for Turing's work we are experiencing it in the immediate of the growing impacts of $\mathrm{Al}$ and machine learning, for da Vinci, we are only beginning to understand his work through its digitisation of his notebooks that provides online access to his enormous corpus of work represented by his codices and notebooks made available over the past few years.

Now 500 years since Leonardo da Vinci's death, his work is being diffused across the Internet inspiring new study and exhibitions of his works, most spectacularly at the da Vinci exhibition at the Louvre in Paris, which reminds us that da Vinci 
spent his last four years, 1515-1519 in France working in service of François I, having brought his most famous painting with him - the Mona Lisa now in the Louvre's permanent collections.

From Leonard da Vinci's notebooks, we see that central to his daily life experience was recording his observations and reflections - that he adhered to the Renaissance principles of drawing and writing about what he was seeing and thinking - and still today, it remains at the heart of knowing. As we compute the future, the knowledge we have inherited from da Vinci and Turing will continue to define our present and future as we enter the age of $\mathrm{Al}$ and machine learning, and a time when the connection of nature and creativity becomes increasingly important.

\section{CONCLUSION}

\section{The Future is Postdigital - New Visions of the Natural World}

As spatial computing becomes more omnipresent in the environment, and wearables digitally capture the body's electrical output of biological systems of the brain, heart, metabolism, etc., we will increasingly see the integration of digital systems in the natural world, for example, embedding digital devices in the body, mediating human experience, making the digital future more natural. Interactive digital wearables that capture biodata from the body's electrical waves and from the spatial computing environment, will make data more interactive and immediate to human life, while tracking ourselves in the environment will heighten our awareness of our emotional states and reactions. "These new kinds of meditation are harnessing the power of your body's own electricity via your wearables to allow the user to feel content in ways that have never been done before." As we move from watching to participating, the human body becomes the mediator while computing plays the enabler (Des Marais 2019).

Over the past decade, digital life seemingly tethered to screens has tended to create a sense of isolation from "real life" (Bowen \& Giannini 2014). By contrast, we enter a new era of human to human interaction enhanced and deepened by invisible computing using Al and machine learning, so that although we will be living with machines on a daily basis, our humanness will be advanced with our ability to share experiences and emotions.

As da Vinci discovered the inner workings of the body and contemplated the human soul, he revealed through his drawings how the body's systems worked and how the human embryo develops into a foetus in the womb, to a human being in the world, while Turing looked inside the mind through neuro science posing the question can computers think, basing his inquiry on his discoveries in artificial intelligence, machine learning and deep neural networks. He spent his last two years of life studying the germination of plants and the systems controlling their patterns of growth. Both men drew upon mathematics and the notion of nature as a unifying life force, although Turing worked in bytes and bits, he shared da Vinci's fascination with the underlying processes that germinate life, whether a plant or a human, as it exists at the levels of cellular, atomic, 1s and 0s all invisible to the naked eye.

Computing our future, will be more and more a process of sharing our existence with robots and thinking with $\mathrm{Al}$ and machine learning, while we are challenged to understand the strengths and weaknesses of human and artificial states of being. By 1950, Turing knew that computational thinking using $\mathrm{Al}$ and machine learning, as demonstrated with the Turing test, that machines could way outstrip humans, but believed that humans in terms of intuition, innovation and creativity, would still hold the lead, although he predicted that advantage would likely be short-lived (Turing 1950). As we move into future, digital time accelerates while digital space contracts the distance between past and present, becoming a new space for the discovery of innovative ideas and ways of thinking.

\section{Acknowledgements}

This paper has taken inspiration from a recent book (Giannini \& Bowen 2019) and previous EVA London papers (Bowen 2016; Bowen et al. 2018; Giannini \& Bowen 2017; 2018). The paper has been written in conjunction with a sister paper for the EVA Florence conference (Bowen \& Giannini 2020). Jonathan Bowen is grateful to Museophile Limited for financial support.

\section{REFERENCES}

ACM (2018) Fathers of the Deep Learning Revolution Receive ACM A.M. Turing Award - Bengio, Hinton and LeCun Ushered in Major Breakthroughs in Artificial Intelligence. https://awards.acm.org/about/2018-turing

Bowen, J. P. (2016) Alan Turing: Virtuosity and visualisation. In Bowen et al. (2016), pp. 197-204. DOI: 10.14236/ewic/EVA2016.40

Bowen, J. P., Diprose, G., and Lambert, N. (eds.) (2016) EVA London 2016: Electronic Visualisation and the Arts. BCS, Electronic Workshops in Computing (eWiC). DOI: 10.14236/ewic/eva2016.0

Bowen, J. P., Diprose, G., and Lambert, N. (eds.) (2017) EVA London 2017: Electronic Visualisation and the Arts. BCS, Electronic Workshops in Computing (eWiC). DOI: $\underline{10.14236 / \text { ewic/eva2017.0 }}$ 
Bowen, J. P. and Giannini, T. (2014) Digitalism: The new realism? In $\mathrm{K}$. Ng, J. P. Bowen, and S. McDaid (eds.), EVA London 2014: Electronic Visualisation and the Arts. BCS, eWiC, pp. 324-331. DOI: 10.14236/ewic/eva2014.38

Bowen, J. P. and Giannini, T. (2020) The Digital Renaissance from da Vinci to Turing. In V. Cappellini et al. (eds.), EVA 2020 Florence Proceedings. DOI: 10.14236/ewic/eva2020.0

Bowen, J. P., Trickett, T., Green, J. B. A., and Lomas, A. (2018) Turing's Genius - Defining an apt microcosm. In Bowen et al. (2018), pp. 155-162. DOI: 10.14236/ewic/EVA2018.31

Bowen, J. P., Weinel, J., Diprose, G., and Lambert, N. (eds.) (2018) EVA London 2018: Electronic Visualisation and the Arts. BCS, Electronic Workshops in Computing (eWiC). DOI: 10.14236/ewic/eva2018.0

Copeland, B. J., Bowen, J. P., Sprevak, M., Wilson, R. J., et al. (2017) The Turing Guide. Oxford University Press. https://en.wikipedia.org/wiki/The Turing Guide

Des Marais, C. (2019) 25 Tech Predictions for 2020. Inc.com, 27 December 2019. https://www.inc.com/ christina-desmarais/25-tech-predictions-for-2020.html

Devlin, K. (2019) The world's first artificial womb for humans. BBC News, BBC, 16 October. https://www.bbc.com/news/av/health-50056405/theworld-s-first-artificial-womb-for-humans

Dowd, V. (2014) What was Alan Turing really like? Witness, BBC World Service. BBC News, BBC, 6 June 2014. https://www.bbc.com/news/magazine-27701207

Giannini, T. and Bowen, J. P. (2017) Life in Code and Digits: When Shannon met Turing. In Bowen et al. (2017), pp. 51-58. DOI: 10.14236/ewic/EVA2017.9

Giannini, T. and Bowen, J. P. (2018) Of Museums and Digital Culture: A landscape view. In Bowen et al. (2018), pp. 172-179. DOI: 10.14236/ewic/EVA2018.34

Giannini, T. and Bowen, J. P. (eds.) (2019) Museums and Digital Culture: New perspectives and research. Springer, Series on Cultural Computing.

Gladwin, L. A. (1997) Alan Turing, Enigma, and the Breaking of German Machine Ciphers in World War II. Turing and Enigma, pp. 203-217. National Archives, USA.

https://www.archives.gov/files/publications/prologue/1997 /fall/turing.pdf
MacCurdy, E. (1955) Notebooks of Leonardo da Vinci. New York: George Braziller. Internet Archive, 2012. https://archive.org/stream/noteboo00leon/noteboo00leon djvu.txt

Manevskava, A. (2017) Reflecting the Invisible or Distorting the Visible: Mirrors in Art. Daily Art Magazine, 19 July. https://www. dailyartmagazine.com/reflecting-theinvisible-or-visible-mirrors-in-art/

Markoff, J. (2011) Computer wins on 'Jeopardy!': Trivial, it's not. The New York Times, USA, 16 February. https://www.nytimes.com/2011/02/17/science/17jeopardy -watson.html

Rharbaoui, Y. (2016) The future Da Vinci is a robot: Al and Artistic Creation. The Medium, 20 November. https://salon.thefamily.co/the-future-da-vinci-is-a-robot-aiand-artistic-creation-51001f12149d

Scienceblocks (2018) Gardening with Alan Turing, and sketching evolution of plants with Da Vinci - Phyllotaxis. Steemit, \#steemstem. https://steemit.com/steemstem/ @scienceblocks/gardening-with-alan-turing-andsketching-evolution-of-plants-with-da-vinci-phyllotaxis3aOfabaacf7c4

Swift, T. (2019) Lover (Lyric Video). YouTube, 15 August.

https://www.youtube.com/watch?v=cvUAzpn48xA

Turing, A. M. (1950) Computing machinery and intelligence. Mind, 59(236), pp. 433-460, October. DOI: 10.1093/mind/LIX.236.433

Turing, A. M. (1951) Can Digital Computers Think? BBC Radio, 15 May 1951. Alan Turing's lost radio broadcast rerecorded. YouTube, 24 December 2017. https://www.youtube.com/watch?v=cMxbSsRntv4

Turing, A. M. (1952) The chemical basis of morphogenesis. Philosophical Transactions of the Royal Society of London, 237(641), pp. 37-72. DOI: 10.1098/rstb.1952.0012

Turing Digital Archive (n.d.) The Turing Digital Archive. Archive Centre, King's College, Cambridge, UK. http://www.turingarchive.org

Weinel, J., Bowen, J. P., Diprose, G., and Lambert, N. (eds.) (2019) EVA London 2019: Electronic Visualisation and the Arts. BCS, Electronic Workshops in Computing (eWiC). ScienceOpen. DOI: 10.14236/ewic/EVA2019.0

Zöllner, F. (2003). Leonardo da Vinci: Complete Paintings and Drawings. Taschen. 